\title{
NEOWISE STUDIES OF ASTEROIDS WITH SLOAN PHOTOMETRY: PRELIMINARY RESULTS
}

\author{
A. Mainzer ${ }^{1}$, J. Masiero ${ }^{1}$, T. Grav ${ }^{2}$, J. Bauer ${ }^{1,3}$, D. J. Tholen ${ }^{4}$, R. S. McMillan ${ }^{5}$, E. Wright ${ }^{6}$, T. Spahr ${ }^{7}$,

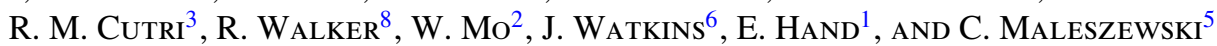 \\ 1 Jet Propulsion Laboratory, California Institute of Technology, Pasadena, CA 91109, USA \\ ${ }^{2}$ Department of Physics and Astronomy, Johns Hopkins University, Baltimore, MD 21218, USA \\ ${ }^{3}$ Infrared Processing and Analysis Center, California Institute of Technology, Pasadena, CA 91125, USA \\ ${ }^{4}$ Institute for Astronomy, University of Hawaii, 2680 Woodlawn Drive, Honolulu, HI 96822-1839, USA \\ ${ }^{5}$ Lunar and Planetary Laboratory, University of Arizona, 1629 East University Blvd., Kuiper Space Science Bldg. \#92, Tucson, AZ 85721-0092, USA \\ ${ }^{6}$ UCLA Division of Astronomy and Astrophysics, P.O. Box 91547, Los Angeles, CA 90095-1547, USA \\ ${ }^{7}$ Minor Planet Center, Harvard-Smithsonian Center for Astrophysics, 60 Garden Street, Cambridge, MA 02138, USA \\ ${ }^{8}$ Monterey Institute for Research in Astronomy, Monterey, CA, USA \\ Received 2011 July 14; accepted 2011 October 3; published 2011 December 27
}

\begin{abstract}
We have combined the NEOWISE and Sloan Digital Sky Survey data to study the albedos of 24,353 asteroids with candidate taxonomic classifications derived using Sloan photometry. We find a wide range of moderate to high albedos for candidate S-type asteroids that are analogous to the $\mathrm{S}$ complex defined by previous spectrophotometrically based taxonomic systems. The candidate C-type asteroids, while generally very dark, have a tail of higher albedos that overlaps the $\mathrm{S}$ types. The albedo distribution for asteroids with a photometrically derived Q classification is extremely similar to those of the S types. Asteroids with similar colors to (4) Vesta have higher albedos than the $\mathrm{S}$ types, and most have orbital elements similar to known Vesta family members. Finally, we show that the relative reflectance at 3.4 and $4.6 \mu \mathrm{m}$ is higher for D-type asteroids and suggest that their red visible and near-infrared spectral slope extends out to these wavelengths. Understanding the relationship between size, albedo, and taxonomic classification is complicated by the fact that the objects with classifications were selected from the visible/near-infrared Sloan Moving Object Catalog, which is biased against fainter asteroids, including those with lower albedos.
\end{abstract}

Key words: atlases - catalogs - infrared: general - minor planets, asteroids: general - surveys

Online-only material: color figures

\section{INTRODUCTION}

Asteroids and comets represent the leftovers from the formation of our solar system. By studying their compositional variation, we can begin to better understand the conditions present at the earliest stages of planet formation as well as their subsequent evolution and processing. Asteroids are grouped into three main categories: $\mathrm{C}$ type or carbonaceous asteroids, thought to be the most common type in the main belt; the $\mathrm{S}$ type or stony asteroids, a spectrally diverse group, and the $\mathrm{X}$ types, another diverse group of asteroids that have relatively featureless spectra but a wide range of albedos, probably representing a broad range of mineralogies and thermal histories. Gaffey et al. (1993) give an overview of some of the earlier taxonomic systems (e.g., Chapman et al. 1975; Bowell et al. 1978; Barucci et al. 1987; Tedesco et al. 1989a, 1989b; Howell et al. 1994). Tholen (1984) created 14 taxonomic classes based on the combinations of colors available from the Eight-Color Asteroid Survey (ECAS; Zellner et al. 1985; Tholen \& Barucci 1989). ECAS used filter passbands with wavelengths ranging from 0.34 to $1.04 \mu \mathrm{m}$, since UV wavelengths were detectable by the photomultiplier available at the time. The Tholen and Tedesco systems also used albedo to differentiate asteroids; the X-group of asteroids have similar ECAS colors but markedly different albedos.

The Tholen and Tedesco systems offer a powerful means of distinguishing unique asteroid groups. However, when CCDs replaced the photomultipliers at most observatories, they ushered in a factor of several improvement in quantum efficiency at red wavelengths compared to blue; while the ECAS photomultipliers extended out to $1.1 \mu \mathrm{m}$, most CCDs' responses were diminished at this wavelength. These effects combined to make it difficult for observers to collect the full set of UV and near-infrared bands used by ECAS. Groups such as Xu et al. (1995), Lazzaro et al. (2004), and Bus \& Binzel (2002a) obtained visible spectra of asteroids and used them for classification without albedos. Bus \& Binzel (2002b) created a new taxonomic scheme relying solely on visible spectroscopy with 26 taxonomic types, and DeMeo et al. (2009) revised that scheme to 24 taxonomic types based on visible and near-infrared (VNIR) spectral signatures. Neither the Bus \& Binzel (2002b) nor DeMeo et al. (2009) systems rely upon having albedo measurements for classification. Nevertheless, systems based solely on VNIR spectroscopy and photometry are widely used because observations at these wavelengths have generally been far more widely available than albedo measurements for the $\sim 500,000$ asteroids known today. However, as discussed in Gaffey et al. (2002), the ability to link taxonomic classification to asteroid mineralogy is complicated by the unknown surface properties such as particle size, the fact that some minerals have limited or no spectral features in the wavelengths used for classification, overlapping features, etc. It is therefore useful to try to understand how the various taxonomic types are linked to physical properties such as albedo and density.

The fourth release of the Sloan Digital Sky Survey (SDSS) Moving Object Catalog (MOC; Stoughton et al. 2002; Abazajian et al. 2003) provided near-simultaneous observations of $\sim 100,000$ known asteroids in five bands $(u, g, r, i$, and $z)$ (Ivezić et al. 2001), and these have been used to study the distribution of colors throughout the main belt (cf. Parker et al. 2008; 

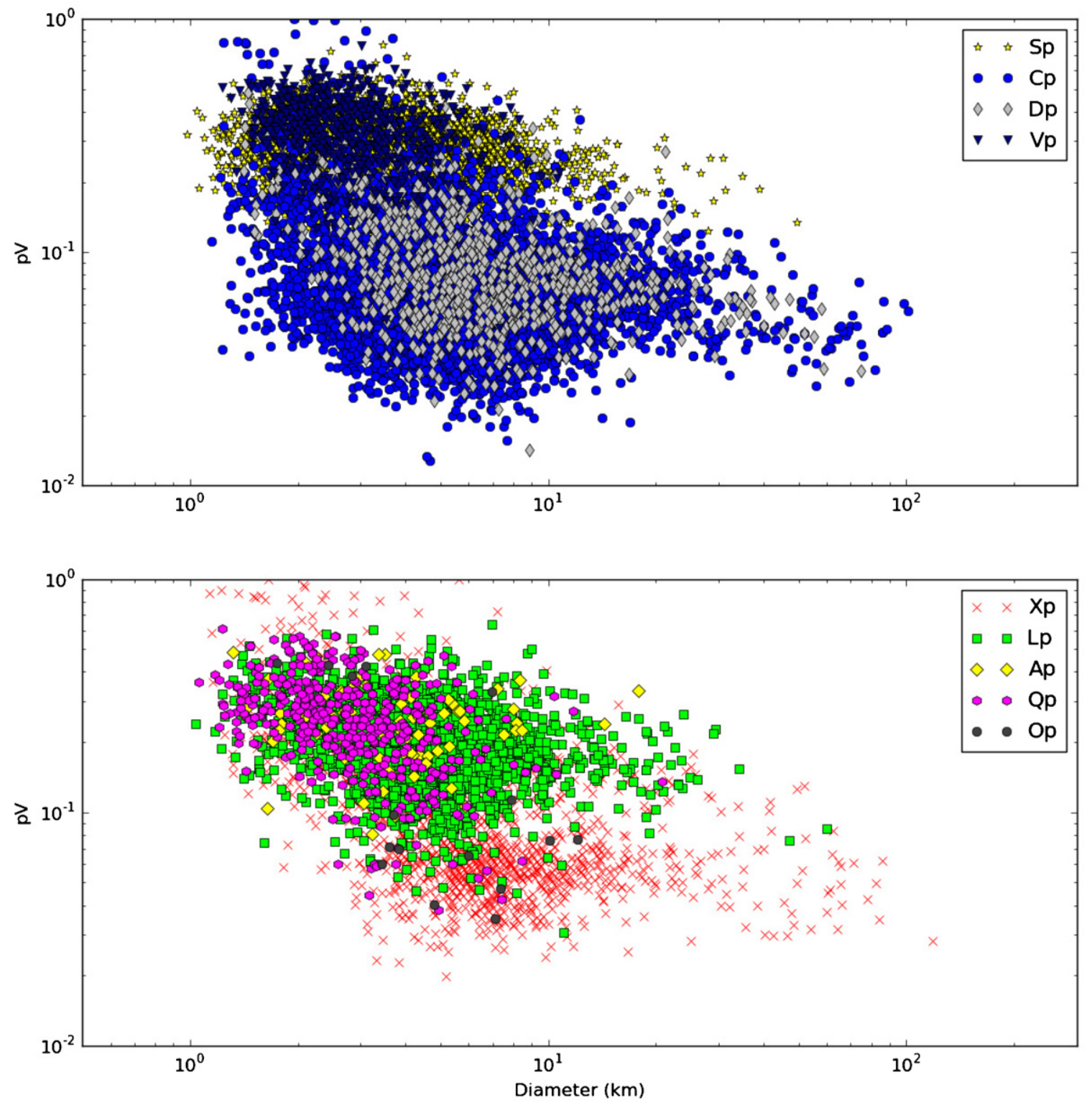

Figure 1. Visible albedo as a function of size for the nine individual classes defined by Carvano et al. (2010). The classes have been separated into two sub-panels for clarity. The bias of the SDSS survey against small, low-albedo objects is evident.

(A color version of this figure is available in the online journal.)

Nesvorný et al. 2005). These studies have been conducted largely without reference to albedo, simply because the number of asteroids in the SDSS MOC vastly outnumbers those with well-measured albedos. To date, the Infrared Astronomical Satellite (Tedesco et al. 2002) has been the largest source of radiometrically measured asteroid albedos, providing measurements for $\sim 2000$ asteroids.

With the Wide-field Infrared Survey Explorer's (WISE) NEOWISE project (Wright et al. 2010; Mainzer et al. 2011a), thermal observations of $>157,000$ asteroids are now in hand. In Mainzer et al. (2011e), we compared the albedos derived from NEOWISE observations of $\sim 1900$ asteroids with taxonomic types derived from VNIR spectroscopy. Here, we use the SDSS MOC photometry to obtain approximate taxonomic classifications for asteroids with NEOWISE observations. We initially focus on the system of Carvano et al. (2010), who define a classification algorithm based on SDSS colors that is compatible with previous taxonomic systems.

\section{OBSERVATIONS}

WISE surveyed the entire sky in four infrared wavelengths, 3.4, 4.6, 12, and $22 \mu \mathrm{m}$ (denoted $W 1, W 2, W 3$, and $W 4$, respectively). Descriptions of the pre-launch mission design and testing can be found in Liu et al. (2008) and Mainzer et al. (2005), and the post-launch description is given in Wright et al. (2010). A series of enhancements to the WISE data processing pipeline, known as NEOWISE, have enabled the detection of $>157,000$ asteroids and comets throughout the solar system, including the discovery of $\sim 34,000$ new minor planets (Mainzer et al. 2011a).

A total of 24,353 objects, including nine NEOs and $~ 24,275$ main belt asteroids (MBAs), were detected during the fully cryogenic portion of the NEOWISE survey and had matches with SDSS MOC observations of sufficient quality to enable classification according to the method described in Carvano et al. (2010). The observations of these objects were extracted from the WISE archive using the First Pass version of the 

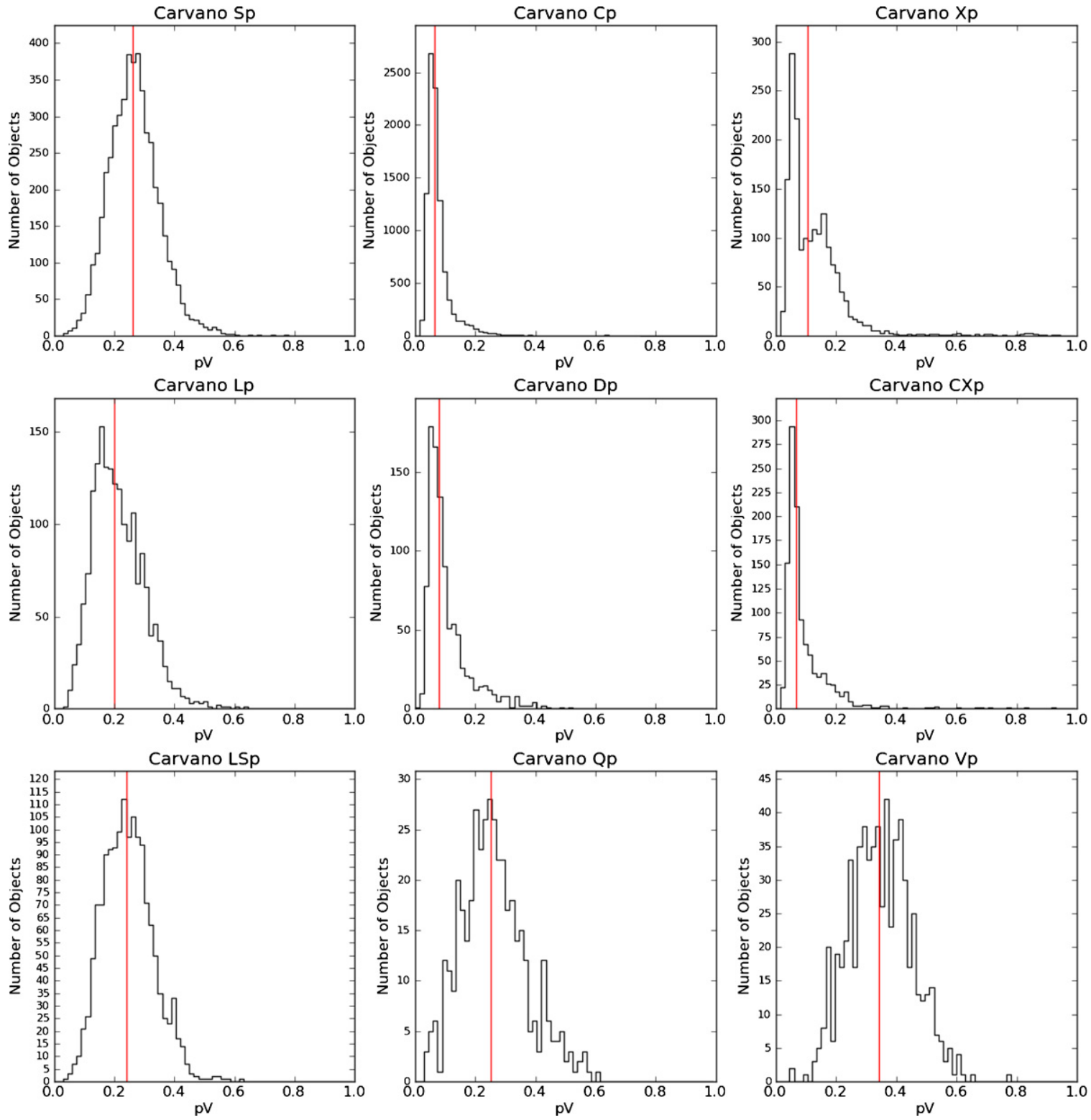

Figure 2. NEOWISE-derived albedos of asteroids observed and classified by Carvano et al. (2010). Only classes with more than $\sim 100$ asteroids are plotted, and these include some objects that have multiple SDSS observations that produced different classifications. The median $p_{V}$ value is shown as a vertical red line.

(A color version of this figure is available in the online journal.)

WISE data processing pipeline (Cutri et al. 2011) following the methods and parameters given in Mainzer et al. (2011c, hereafter M2), Mainzer et al. (2011d, M3), and Mainzer et al. (2011e, M4).

\section{PRELIMINARY THERMAL MODELING}

We have created preliminary thermal models for each asteroid using the First-Pass Data Processing Pipeline described above. As described in references M2, M3, and M4, we employ the near-Earth asteroid thermal model (NEATM) of Harris (1998). The NEATM model uses the so-called beaming parameter $\eta$ to account for cases intermediate between zero thermal inertia (Lebofsky \& Spencer 1989, the Standard Thermal Model) and high thermal inertia (Lebofsky et al. 1978, the Fast Rotating Model; Veeder et al. 1989, the Fast Rotating Model; Lebofsky \& Spencer 1989, the Fast Rotating Model). With NEATM, $\eta$ is a free parameter that can be fit when two or more infrared bands are available. Bands $W 1$ and $W 2$ typically contain a mix of reflected sunlight and thermal emission. The flux from reflected sunlight was computed for each WISE band using the methods described in M2, M3, and M4; when sufficient reflected sunlight was present in bands $W 1$ and $W 2$, it was possible to compute the reflectivity at these wavelengths, $p_{\mathrm{IR}}$, where we make the assumption that $p_{\mathrm{IR}}=p_{3.4}=p_{4.6}$. The validity of this assumption and the meaning of $p_{\mathrm{IR}}$ is discussed in M4 and will be the subject of future work. As described in M2 and M3, the minimum diameter error that can be achieved using WISE observations is $\sim 10 \%$, and the minimum relative albedo error is $\sim 20 \%$ for objects with more than one WISE thermal band for which $\eta$ can be fitted. For objects with large amplitude light curves, poor $H$ measurements, or poor signal-to-noise measurements in the WISE bands, the errors will be higher. 
Median Values of $p_{V}$ and $p_{\mathrm{IR}} / p_{V}$ using NEOWISE Cryogenic Observations of Asteroids with Taxonomic Types Derived from SDSS Colors

\begin{tabular}{|c|c|c|c|c|c|c|c|c|c|c|c|c|c|c|c|c|}
\hline Class & $\begin{array}{c}N \\
p_{V} \\
\end{array}$ & $\begin{array}{c}\text { Median } \\
p_{V} \\
\end{array}$ & SD & Min & Max & $\begin{array}{c}N \\
p_{\text {IR }} / p_{V} \\
\end{array}$ & $\begin{array}{l}\text { Median } \\
p_{\mathrm{IR}} / p_{V}\end{array}$ & $\begin{array}{l}\text { SD } \\
\text { SD }\end{array}$ & $\begin{array}{l}\text { Min } \\
\text { Min }\end{array}$ & $\begin{array}{l}\text { Max } \\
\text { Max }\end{array}$ & $\begin{array}{c}N \\
p_{V} 50 \\
\end{array}$ & $\begin{array}{c}\text { Median } \\
p_{V} 50 \\
\end{array}$ & $\begin{array}{l}\text { SD } \\
50 \\
\end{array}$ & $\begin{array}{c}N 50 \\
p_{\mathrm{IR}} / p_{V}\end{array}$ & $\begin{array}{c}\text { Median } 50 \\
p_{\mathrm{IR}} / p_{V} \\
\end{array}$ & $\begin{array}{l}\text { SD } \\
50 \\
\end{array}$ \\
\hline $\mathrm{Sp}$ & 4880 & $0.262 \pm 0.001$ & 0.084 & 0.034 & 0.779 & 474 & $1.452 \pm 0.028$ & 0.603 & 0.607 & 4.945 & 2725 & $0.267 \pm 0.002$ & 0.080 & 362 & $1.449 \pm 0.030$ & 0.570 \\
\hline $\mathrm{Cp}$ & 9779 & $0.064 \pm 0.001$ & 0.055 & 0.013 & 1.000 & 566 & $1.147 \pm 0.032$ & 0.765 & 0.260 & 4.971 & 3069 & $0.067 \pm 0.001$ & 0.054 & 374 & $1.095 \pm 0.035$ & 0.680 \\
\hline $\mathrm{Xp}$ & 1773 & $0.106 \pm 0.003$ & 0.118 & 0.020 & 1.000 & 201 & $1.318 \pm 0.044$ & 0.625 & 0.507 & 4.801 & 502 & $0.109 \pm 0.006$ & 0.127 & 138 & $1.322 \pm 0.056$ & 0.658 \\
\hline Lp & 1838 & $0.202 \pm 0.002$ & 0.085 & 0.030 & 0.638 & 155 & $1.263 \pm 0.047$ & 0.580 & 0.577 & 4.036 & 768 & $0.204 \pm 0.003$ & 0.087 & 109 & $1.252 \pm 0.050$ & 0.518 \\
\hline $\mathrm{Dp}$ & 984 & $0.080 \pm 0.002$ & 0.073 & 0.014 & 0.516 & 104 & $2.079 \pm 0.073$ & 0.744 & 1.034 & 4.543 & 235 & $0.073 \pm 0.003$ & 0.041 & 67 & $2.165 \pm 0.094$ & 0.766 \\
\hline Ap & 85 & $0.248 \pm 0.009$ & 0.087 & 0.081 & 0.488 & 9 & $2.526 \pm 0.212$ & 0.637 & 1.307 & 3.166 & 20 & $0.273 \pm 0.018$ & 0.082 & 4 & $2.187 \pm 0.262$ & 0.523 \\
\hline Qp & 424 & $0.253 \pm 0.005$ & 0.110 & 0.038 & 0.613 & 14 & $1.456 \pm 0.106$ & 0.398 & 0.939 & 2.144 & 118 & $0.295 \pm 0.009$ & 0.093 & 7 & $1.100 \pm 0.172$ & 0.455 \\
\hline Op & 16 & $0.076 \pm 0.039$ & 0.157 & 0.035 & 0.436 & 0 & $0.000 \pm 0.000$ & 0.000 & 0.000 & 0.000 & 0 & $0.000 \pm 0.000$ & 0.000 & 0 & $0.000 \pm 0.000$ & 0.000 \\
\hline $\mathrm{Vp}$ & 650 & $0.343 \pm 0.004$ & 0.105 & 0.047 & 0.771 & 47 & $1.470 \pm 0.092$ & 0.631 & 0.886 & 4.046 & 288 & $0.352 \pm 0.006$ & 0.100 & 29 & $1.560 \pm 0.099$ & 0.532 \\
\hline $\mathrm{CSp}$ & 1 & $0.130 \pm 0.000$ & 0.000 & 0.130 & 0.130 & 0 & $0.000 \pm 0.000$ & 0.000 & 0.000 & 0.000 & 0 & $0.000 \pm 0.000$ & 0.000 & 0 & $0.000 \pm 0.000$ & 0.000 \\
\hline XSp & 36 & $0.203 \pm 0.017$ & 0.100 & 0.044 & 0.507 & 2 & $0.885 \pm 0.040$ & 0.056 & 0.829 & 0.941 & 1 & $0.507 \pm 0.000$ & 0.000 & 1 & $0.829 \pm 0.000$ & 0.000 \\
\hline LSp & 1488 & $0.240 \pm 0.002$ & 0.083 & 0.042 & 0.623 & 124 & $1.476 \pm 0.043$ & 0.479 & 0.673 & 3.472 & 396 & $0.247 \pm 0.004$ & 0.083 & 79 & $1.449 \pm 0.054$ & 0.480 \\
\hline SQp & 453 & $0.252 \pm 0.004$ & 0.092 & 0.062 & 0.617 & 17 & $1.561 \pm 0.117$ & 0.484 & 1.069 & 3.085 & 93 & $0.262 \pm 0.009$ & 0.084 & 8 & $1.444 \pm 0.098$ & 0.276 \\
\hline SAp & 31 & $0.273 \pm 0.016$ & 0.091 & 0.135 & 0.451 & 3 & $1.672 \pm 0.125$ & 0.216 & 1.299 & 1.811 & 0 & $0.000 \pm 0.000$ & 0.000 & 0 & $0.000 \pm 0.000$ & 0.000 \\
\hline SVp & 44 & $0.316 \pm 0.015$ & 0.097 & 0.111 & 0.549 & 2 & $1.874 \pm 0.407$ & 0.575 & 1.299 & 2.450 & 0 & $0.000 \pm 0.000$ & 0.000 & 0 & $0.000 \pm 0.000$ & 0.000 \\
\hline CXp & 1144 & $0.067 \pm 0.003$ & 0.087 & 0.020 & 0.999 & 67 & $1.243 \pm 0.104$ & 0.851 & 0.643 & 5.031 & 104 & $0.069 \pm 0.011$ & 0.110 & 20 & $1.122 \pm 0.062$ & 0.276 \\
\hline CDp & 22 & $0.064 \pm 0.012$ & 0.057 & 0.030 & 0.240 & 0 & $0.000 \pm 0.000$ & 0.000 & 0.000 & 0.000 & 0 & $0.000 \pm 0.000$ & 0.000 & 0 & $0.000 \pm 0.000$ & 0.000 \\
\hline CQp & 6 & $0.139 \pm 0.017$ & 0.042 & 0.083 & 0.192 & 0 & $0.000 \pm 0.000$ & 0.000 & 0.000 & 0.000 & 0 & $0.000 \pm 0.000$ & 0.000 & 0 & $0.000 \pm 0.000$ & 0.000 \\
\hline XLp & 170 & $0.159 \pm 0.005$ & 0.060 & 0.027 & 0.452 & 10 & $1.116 \pm 0.122$ & 0.387 & 0.491 & 2.015 & 26 & $0.160 \pm 0.016$ & 0.081 & 5 & $1.059 \pm 0.099$ & 0.221 \\
\hline XDp & 209 & $0.076 \pm 0.006$ & 0.091 & 0.018 & 0.715 & 11 & $1.628 \pm 0.172$ & 0.569 & 0.996 & 2.689 & 0 & $0.000 \pm 0.000$ & 0.000 & 0 & $0.000 \pm 0.000$ & 0.000 \\
\hline DLp & 170 & $0.178 \pm 0.007$ & 0.091 & 0.044 & 0.531 & 9 & $1.610 \pm 0.148$ & 0.445 & 1.062 & 2.456 & 2 & $0.214 \pm 0.085$ & 0.120 & 0 & $0.000 \pm 0.000$ & 0.000 \\
\hline QVp & 40 & $0.352 \pm 0.015$ & 0.097 & 0.088 & 0.575 & 1 & $1.488 \pm 0.000$ & 0.000 & 1.488 & 1.488 & 0 & $0.000 \pm 0.000$ & 0.000 & 0 & $0.000 \pm 0.000$ & 0.000 \\
\hline QLp & 5 & $0.249 \pm 0.031$ & 0.069 & 0.145 & 0.361 & 0 & $0.000 \pm 0.000$ & 0.000 & 0.000 & 0.000 & 0 & $0.000 \pm 0.000$ & 0.000 & 0 & $0.000 \pm 0.000$ & 0.000 \\
\hline
\end{tabular}

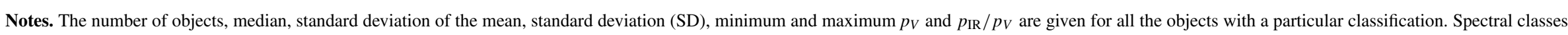

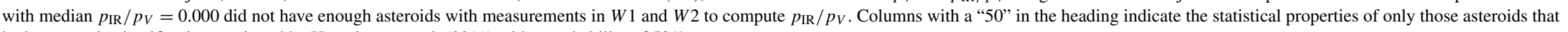
had taxonomic classifications assigned by Hasselmann et al. (2011) with a probability of 50\% or greater. 

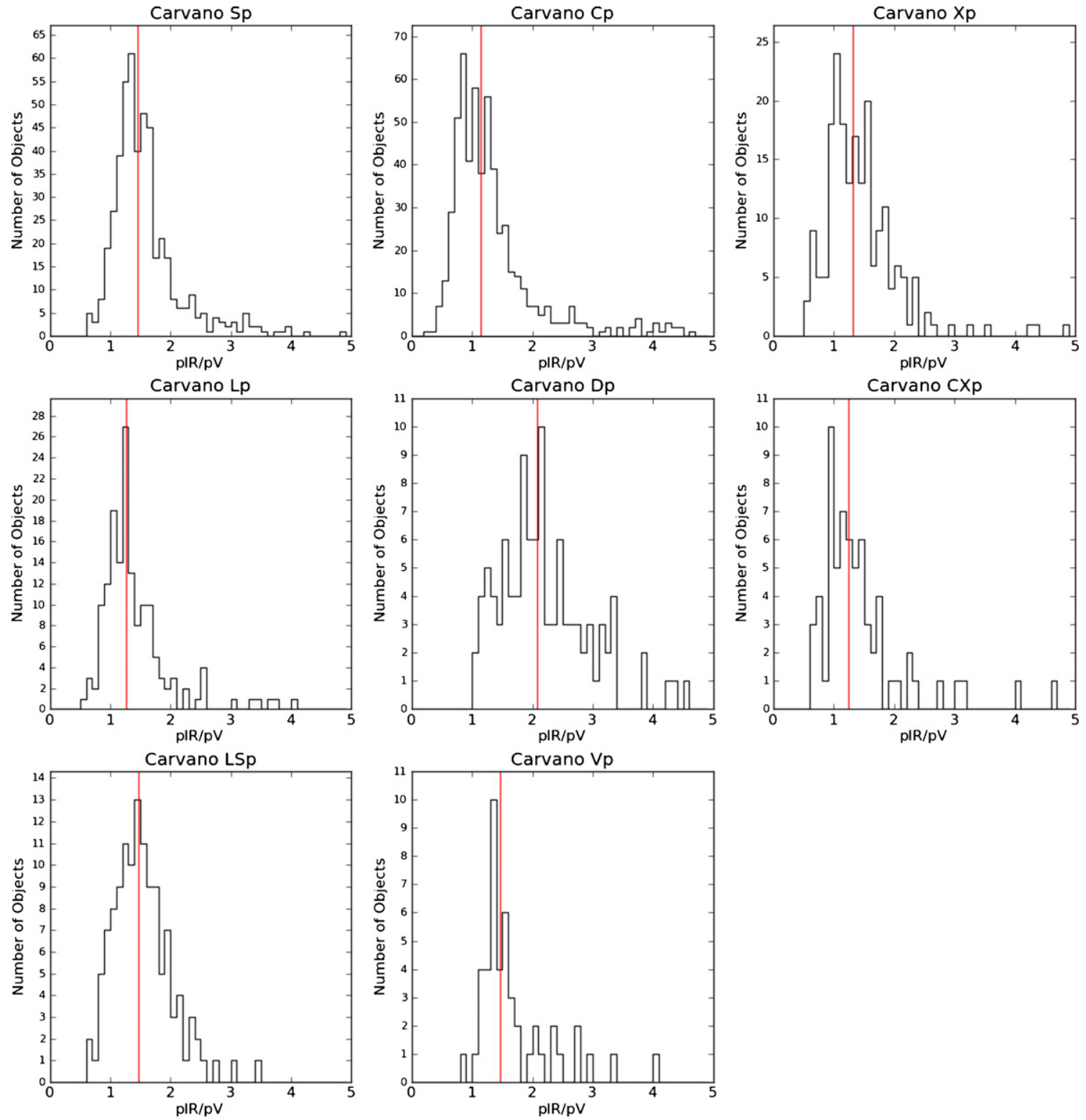

Figure 3. NEOWISE-derived ratio $p_{\mathrm{IR}} / p_{V}$ for asteroids observed and classified according to the system of Carvano et al. (2010). Only asteroids for which $p_{\mathrm{IR}} / p_{V}$ could be fitted are included in this plot. While the $C_{p}$ and $D_{p}$ classes have similar $p_{V}$ values, $p_{\mathrm{IR}} / p_{V}$ is distinctly different. The median $p_{\mathrm{IR}} / p_{V}$ value is shown as a vertical red line.

(A color version of this figure is available in the online journal.)

\subsection{High-albedo Objects}

We note that among the 24,353 asteroids considered here, there are $\sim 55$ that have $p_{V}>0.65$. Of these, 48 have $W 3$ peakto-peak variations $>0.3 \mathrm{mag}$, indicating that they are likely to be highly elongated. Almost all of the extremely high-albedo objects have orbital elements consistent with membership in either the Vesta family or the Hungarias. Harris \& Young (1988) and Harris et al. (1989) noted that E- and V-type asteroids can have slope values as high as $G \sim 0.5$. The assumption that we have used of $G=0.15$ for an object like this would cause an error in the computed $H$ for observations at $20^{\circ}$ phase angle of $\sim 0.3 \mathrm{mag}$; this would drive the albedo derived using such an $H$ value up by 0.3 . These objects would greatly benefit from an improved determination of their $H$ and $G$ values.

\section{DISCUSSION}

The Carvano scheme uses the SDSS colors as well as their measurement uncertainties to define nine spectral classes: $V_{p}, O_{p}, S_{p}, A_{p}, \mathcal{L}_{p}, D_{p}, X_{p}, Q_{p}$, and $C_{p}$. These are roughly analogous to the spectroscopically defined systems of Bus \& Binzel (2002b) and DeMeo et al. (2009); the " $p$ " indicates that the classification was derived photometrically. The nine Carvano classes were defined based on the ability of the SDSS colors to represent the system of Bus \& Binzel (2002b). The exception to this is the $\mathcal{L}_{p}$ class, which is a conglomeration of the Bus K, L, and Ld classes. For each SDSS observation, the probability that an object could be associated with a particular class was computed using the five SDSS magnitudes and their associated uncertainties; classifications and probabilities for $\sim 63,000$ asteroids were taken from Hasselmann et al. (2011). 
Figure 1 shows $p_{V}$ as a function of diameter for the individual Carvano classes. The bias of the VNIR SDSS survey against small, low-albedo asteroids is evident in the dearth of objects in this regime, despite the fact that low-albedo objects dominate most of the main belt (Masiero et al. 2011). As discussed in M4, this bias complicates study of the relationship between size and $p_{V}$; this relationship is best studied using the full NEOWISE data set as it is relatively unbiased with respect to $p_{V}$. Figure 2 shows histograms of $p_{V}$ for the various taxonomic types, and Table 1 summarizes their statistical properties. The $S_{p}$ types are fairly well grouped but span a wide range of moderate to high $p_{V}$; while the $C_{p}$ types tend to have significantly lower $p_{V}$, roughly $15 \%$ have $p_{V}>0.1$, and the $D_{p}$ types have $36 \%$ with $p_{V}$ $>0.1$. In M4, we found that spectroscopically classified $\mathrm{C}$ types with diameters $>30 \mathrm{~km}$ tended to have $p_{V}<0.1$. The fact that so many $C_{p}$ and $D_{p}$ types with diameters $<30 \mathrm{~km}$ have higher albedos suggests either that it is difficult to distinguish these objects with the SDSS colors in the Carvano scheme, that the bias against small objects with low $p_{V}$ is skewing the result, or there is real scatter within the population of $C_{p}$ and $D_{p}$ types. The bias in the SDSS observations against small, low-albedo objects means that measured $p_{V}$ distributions will be skewed higher. Table 1 gives the statistical properties of the various classes both for all objects and for objects with classifications assigned with probabilities $>50 \%$. As can be seen in the table, removing the objects with low probabilities does not significantly affect the results.

Some asteroids have multiple observations in SDSS, and the different SDSS observations can each result in different classifications. Out of the 24,353 asteroids, 3924 received multiple classifications. As described in Carvano et al. (2010), the most frequent variations occur between classes with adjacent limits, such as $\mathcal{L}_{p}$ and $S_{p}$, and from $C_{p}$ to $X_{p}$. As can be observed in Figure 2, the $\mathcal{L} S_{p}$ objects have an albedo distribution that resembles a blend of $S_{p}$ and $\mathcal{L}_{p}$ types; similarly, the $\mathrm{C} X_{p}$ types' albedos appear to be a cross between $C_{p}$ and $X_{p}$ types. Of more interest are the objects with classifications that span dissimilar classes, such as $C_{p}$ to $S_{p}$ or $C_{p}$ to $Q_{p}$; however, only a handful of objects in our sample fall into these classes. VNIR spectroscopy of these objects would illuminate what combination of slopes and/or absorption features gives rise to their diverse set of albedos.

$C_{p}$ and $D_{p}$ types. As discussed in $\mathrm{M} 4$, the B, C, D, and T types of Bus \& Binzel (2002b) and Tholen (1984) have similarly low $p_{V}$, yet they are distinguished by markedly different $p_{\mathrm{IR}} / p_{V}$. We observe a similar phenomenon here with the $C_{p}$ and $D_{p}$ types. Figure 3 shows the distribution of $p_{\mathrm{IR}} / p_{V}$ for those asteroids with sufficient reflected light in $W 1$ or $W 2$ to compute it. The $C_{p}$ and $D_{p}$ types have median $p_{V}=0.064 \pm 0.001$ and $0.080 \pm$ 0.002 , respectively, yet the $C_{p}$ types have $p_{\mathrm{IR}} / p_{V}=$ $1.147 \pm 0.032 ; D_{p}$ types have $p_{\mathrm{IR}} / p_{V}=2.079 \pm 0.073$. In M4, we hypothesized that the divergent $p_{\mathrm{IR}} / p_{V}$ was caused by the fact that the $C_{p}$ types' relatively flat spectra from 0.4 to $1.0 \mu \mathrm{m}$ extends out to 3-4 $\mu \mathrm{m}$, while the $D_{p}$ types slope steeply redward in VNIR wavelengths, continuing out to the $W 1$ and $W 2$ bands. Figure 4 illustrates the utility of $p_{\mathrm{IR}} / p_{V}$ in distinguishing various "dark" asteroid types from one another. We have made the assumption that $p_{W 1}=p_{W 2}$ at present and will revisit this assumption in the future. As discussed above, although the $C_{p}$ and $D_{p}$ asteroids generally have low albedos, they have a tail of higher albedos that are more similar to the bulk of the S-complex objects. If we consider only those asteroids with classification probabilities given by Hasselmann et al. (2011) as being greater

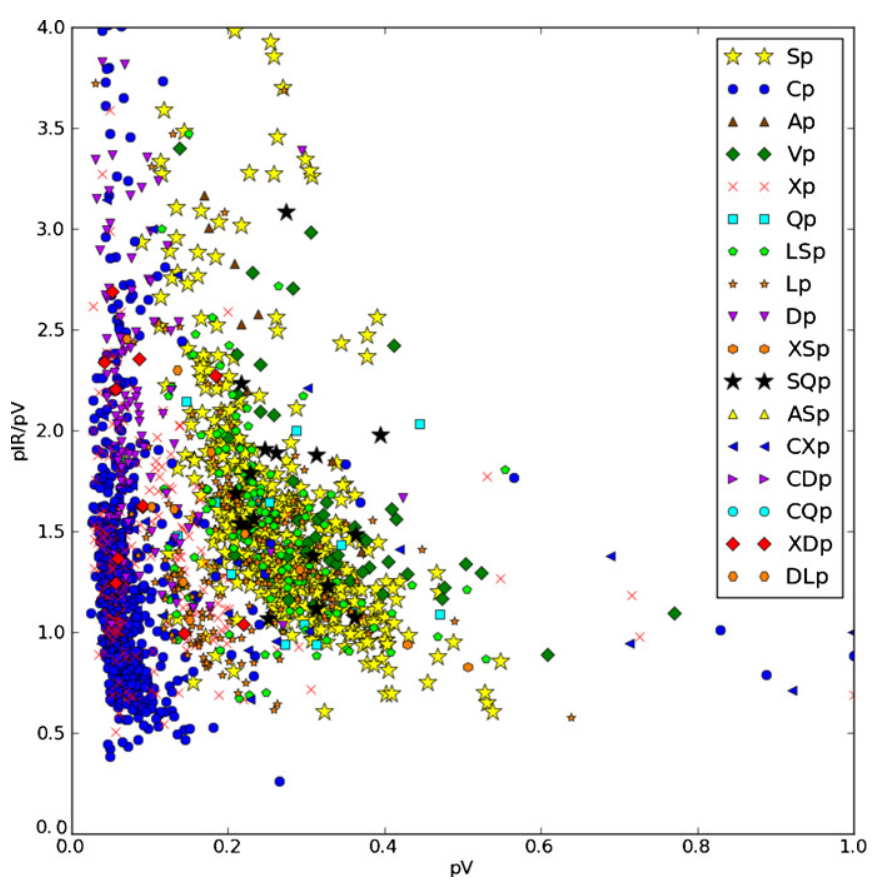

Figure 4. NEOWISE-derived ratio $p_{\mathrm{IR}} / p_{V}$ vs. $p_{V}$ for asteroids observed and classified according to the Carvano taxonomic classification scheme. Only the $\sim 1800$ asteroids for which $p_{\mathrm{IR}} / p_{V}$ could be fitted are included in this plot. (A color version of this figure is available in the online journal.)

than $50 \%$, the tail of high-albedo objects among the $C_{p}$ and $D_{p}$ types does not disappear. This tail of high albedo objects could either represent real scatter within the populations' albedos, or it could be that it is somewhat difficult to classify $C$ and $D$ types based on their SDSS photometry alone. Also, selection biases in the SDSS catalog mean that SDSS was more likely to have detected higher albedo asteroids, even among the $C$ and $D$ types.

$X$ complex. The distribution of NEOWISE-derived albedos found for $X_{p}$ asteroids closely resembles that observed in the general, undebiased main belt population (Masiero et al. 2011). We observed a similarly wide range of albedos in M4 for asteroids spectroscopically classified as X complex. The wide range of albedos is not surprising, since the Tholen $\mathrm{X}$ type (from which the Bus and Bus-DeMeo X types are derived) consists of $\mathrm{E}, \mathrm{M}$, and $\mathrm{P}$ asteroids that are separated by their albedos. The Carvano $X_{p}$ type is based upon the Bus and Bus-DeMeo $\mathrm{X}$ types, neither of which use $p_{V}$ as part of their classification schemes.

$S_{p}, \mathcal{L}_{p}, A_{p}$, and $Q_{p}$ types. Bus \& Binzel (2002b) deemed the $S$ complex to have sufficient spectroscopic variation to warrant dividing it into six different subclasses. With the five SDSS bands, the Carvano system does not separate the $\mathrm{S}$ complex. The $S_{p}$ objects span a wide range of albedos with a similar though slightly higher mean value than the $\mathcal{L}_{p}$ types. Their $p_{\mathrm{IR}} / p_{V}$ values largely overlap, though the peak of the distribution shown in Figure 3 is slightly lower for the $\mathcal{L}_{p}$ types. The $\mathcal{L}_{p}$ type is an amalgamation of the Bus $\mathrm{K}, \mathrm{L}$, and Ld classes, and these are distinguished by the degree of reddening shortward of the $i^{\prime}$ band and flattening longward of $\sim 1 \mu \mathrm{m}$. The $A_{p}$ types are thought to represent mantle material, and for the 85 objects we observed with NEOWISE, we find that their albedos are very similar to the $S_{p}$ types. Bus \& Binzel (2002b) interpret Q-type asteroids as being the un-spaceweathered parent bodies of the ordinary chondrite meteorites; see Chapman (2004) for a discussion of the effects of space weathering. In M4, we found NEOWISE observations of a 

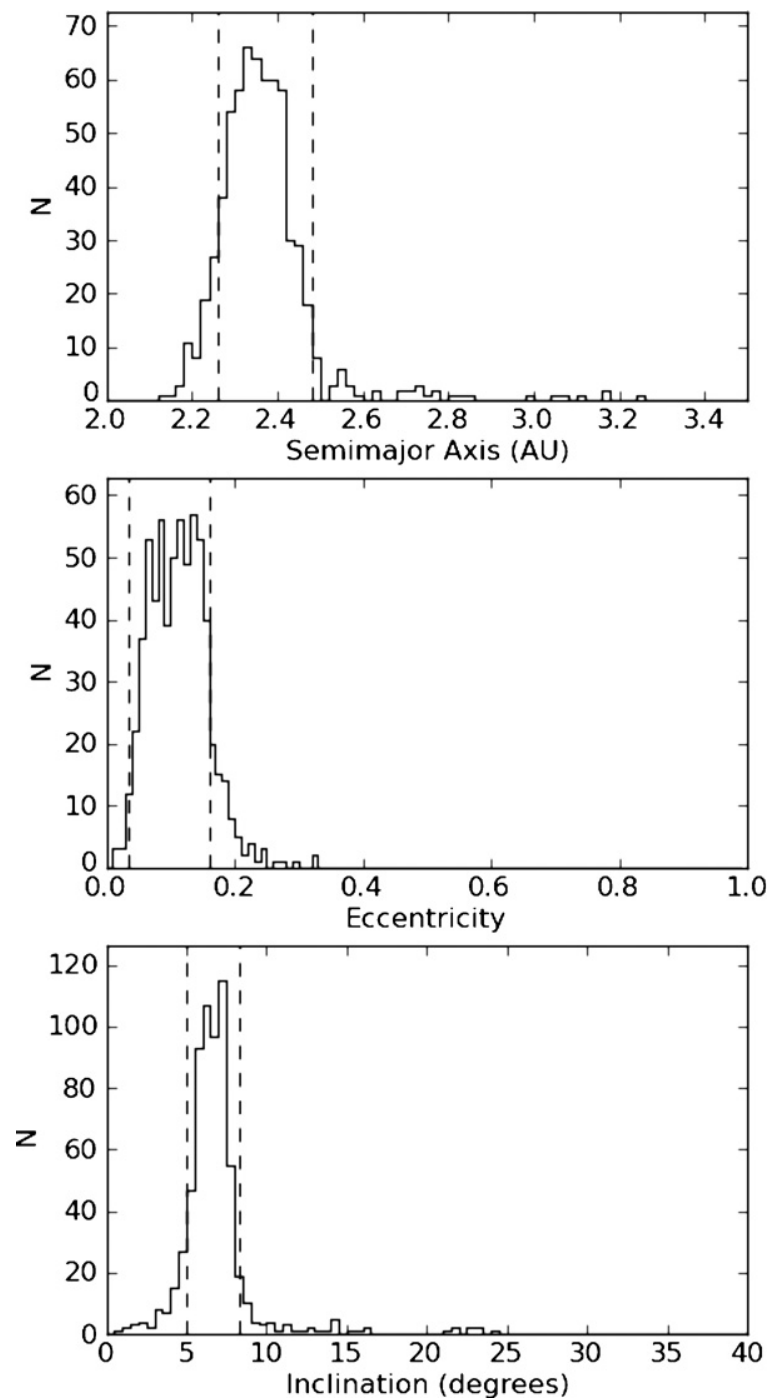

Figure 5. Orbital elements of the 650 asteroids classified as $V_{p}$; the typical ranges for the Vesta family are shown as dashed vertical lines (Nesvorný 2010).
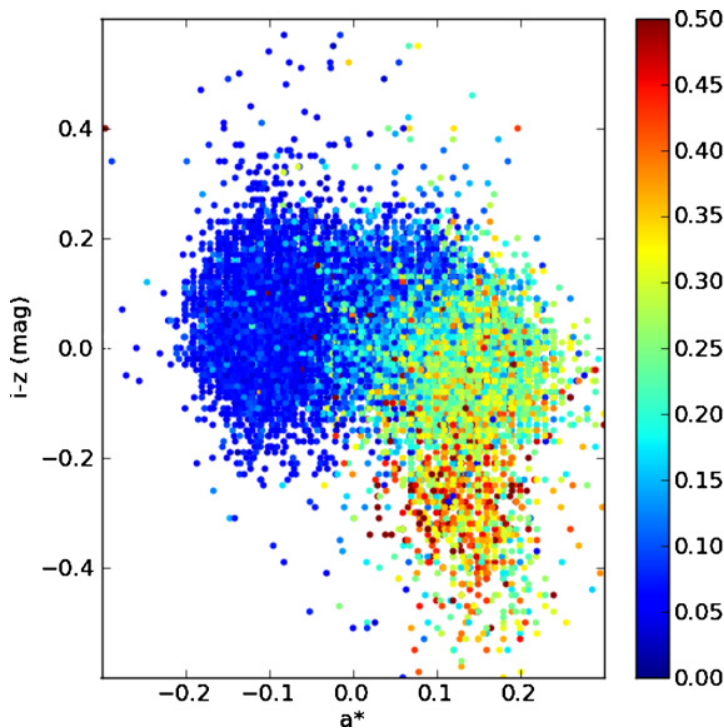

handful of objects spectroscopically determined to be Q types, but their albedos were identical to the $S$ complex; further, we found no significant differences between the DeMeo et al. (2009) $\mathrm{S}$ and $\mathrm{Sw}$ types, where the $\mathrm{Sw}$ type is thought to represent weathered $\mathrm{S}$ types. Here, we find $424 Q_{p}$ types with cryogenic NEOWISE observations sufficient to determine albedo, and our results reveal that the $Q_{p}$ types have slightly lower $p_{V}$ than the $S_{p}$ types. All but 2 of the $424 Q_{p}$ types are MBAs or Mars crossers, and they are distributed throughout the main belt as shown in Carvano et al. (2010); it would be worthwhile to obtain VNIR spectroscopy of these objects in order to determine whether or not the $\mathrm{Q}$ classification is accurate as the main belt is generally thought to be bereft of Q types (Binzel et al. 2010). A nearly equal number of asteroids (453) are classified as being intermediate in type between $S_{p}$ and $Q_{p}$ with extremely similar $p_{V}$.

$O_{p}$ asteroids. Binzel et al. (1993) identified (3628) Božněmcová as the possible source of the L6 and LL6 ordinary chondrites based on the similarity of its reflectance spectrum to laboratory measurements of these common meteorites. Based on its spectral similarity to (1862) Apollo, they use Apollo's albedo and slope parameter $G$ to compute a diameter of $7 \mathrm{~km}$. From NEOWISE observations of (3628) Božněmcová, we compute a diameter of $7.0 \pm 0.2 \mathrm{~km}$ with an albedo of $0.330 \pm 0.043$. Only two asteroids classified as O-type spectroscopically according to the taxonomic system of Bus \& Binzel (2002b) were observed by NEOWISE, (3628) Božněmcová and (169) Zelia, which is classified as an O type by Lazzaro et al. (2004) using the taxonomic system of Bus \& Binzel (2002b). Using NEOWISE observations, Zelia has a diameter of $38.6 \pm 0.9 \mathrm{~km}$ and an albedo of $0.178 \pm 0.035$; its ratio $p_{\mathrm{IR}} / p_{V}=2.08 \pm 0.32$. Carvano et al. (2010) identified 63 asteroids as O-type candidates, and we have observed 16 with NEOWISE, including Božněmcová. As shown in Table 1, these objects span a wide range of albedos, from 0.035 to 0.436 , with a median value of 0.076 ; the largest is $12.1 \pm 0.2 \mathrm{~km}$ in diameter, and the smallest is $1.75 \pm 0.23 \mathrm{~km}$. None of these had measurements in $W 1$ or $W 2$, so $p_{\mathrm{IR}} / p_{V}$ could not be determined. It would be worth obtaining spectroscopic follow-up of these O-type candidates (asteroids 11753,

Figure 6. SDSS a* vs. $i-z$ band magnitudes, with albedo shown as colored points; the shading of C- and S-type points appears as expected from Ivezić et al. (2001, 2002).

(A color version of this figure is available in the online journal.) 
20477, 21692, 29540, 32846, 34341, 57736, 104596, 118595, $140949,164099,178653,247450,261454,2005$ VO101) to see if their spectra confirm their SDSS-based classifications. We note that the $O_{p}$ types with the highest albedos also have the highest probabilities assigned by Hasselmann et al. (2011); all of the $O_{p}$ types with $p_{V}<0.2$ have probabilities lower than $25 \%$.

$V_{p}$ type. Carvano et al. (2010) found a clustering of $V_{p}$-type asteroids near the Vesta family, with some scattered objects throughout the rest of the main belt. We observed $650 V_{p}$ objects with NEOWISE and find that they consistently have high $p_{V}$, tending to be brighter than the $S_{p}$ types. Since the $V_{p}$ types occupy a distinct region of albedo space compared to the background MBAs (Masiero et al. 2011), it may be possible to use albedo in conjunction with orbital elements to find new candidate members of the Vesta family; this will be explored in a future work. The orbital elements of these objects are consistent with Vesta family members identified by Nesvorný (2010), with a median semimajor axis of $2.35 \mathrm{AU}$ and standard deviation (SD) of $0.13 \mathrm{AU}$; median eccentricity of 0.11 with $\mathrm{SD}=0.04$; and median inclination of 6.5 with $\mathrm{SD}=2.4$ (Figure 5). It would be desirable to obtain VNIR spectra of the $V_{p}$ asteroids identified herein to further explore their true natures. Following the work of Ivezić et al. (2001, 2002), we have plotted SDSS a* vs. $i-z$ band magnitudes, with albedo shown as colored points (Figure 6). As noted by Jurić et al. (2002), asteroids with the bluest $i-z$ colors appear to be associated with the Vesta family, and it can be seen in Figure 6 that these objects tend to have high visible albedos.

\section{CONCLUSIONS}

By examining the intersection between the large thermal infrared NEOWISE data set and the VNIR SDSS observations, we have further explored the relationship between taxonomic classifications and $p_{V}$ for 24,353 asteroids and at $3-4 \mu \mathrm{m}$ for 1819 asteroids. We find that the Carvano $S_{p}$ and $V_{p}$ types appear to be relatively robust in identifying asteroids with high $p_{V}$. While the $C_{p}$ types are generally significantly darker, we observe in the $C_{p}$ and $D_{p}$ populations high-albedo tails that lead us to conclude that albedo cannot be conclusively determined purely from photometric VNIR taxonomy. We find a number of candidate O-type asteroids among our sample; others have suggested that these are the progenitors of the LL6 ordinary chondrites, as well as Q-type candidates throughout the main belt. The objects with SDSS colors consistent with V-type asteroids typically have very high albedos, and their orbital elements are similar to known Vesta family members, suggesting that these new objects may indeed be family members as well. As in Mainzer et al. (2011e), we find that $D_{p}$ types, which have a steeply red VNIR spectrum, also have systematically larger $p_{\mathrm{IR}} / p_{V}$ than $C_{p}$ types, despite having similar $p_{V}$ distributions. These higher $p_{\mathrm{IR}} / p_{V}$ values suggest that the $D_{p}$ types' red spectrum continues through the WISE $W 1$ and $W 2$ bands at $3-4 \mu \mathrm{m}$. We caution that since these taxonomic types are determined from objects selected by a visible survey, the population is biased against low-albedo objects, and hence the albedo distributions we have determined are similarly biased, particularly at the smallest size scales. This bias could be mitigated by obtaining a spectrally classified sample that better represents the true population of asteroids, both bright and dark. Such a sample could be created by choosing a set of objects from the NEOWISE survey, which has been found to be essentially unbiased with respect to $p_{V}$ (Mainzer et al. 2011b), and obtaining taxonomic classifications for these objects using a combination of literature values and new observations. While painstaking, such an effort would allow the distinction between observational bias and the physical links between size, albedo, and taxonomic classification to be clarified.

This publication makes use of data products from the Widefield Infrared Survey Explorer, which is a joint project of the University of California, Los Angeles, and the Jet Propulsion Laboratory/California Institute of Technology, funded by the National Aeronautics and Space Administration. This publication also makes use of data products from NEOWISE, which is a project of the Jet Propulsion Laboratory/California Institute of Technology, funded by the Planetary Science Division of the National Aeronautics and Space Administration. We thank our referee, Dr. Schelte J. Bus, for his comments, which have greatly improved this paper. We gratefully acknowledge the extraordinary services specific to NEOWISE contributed by the International Astronomical Union's Minor Planet Center, operated by the Harvard-Smithsonian Center for Astrophysics, and the Central Bureau for Astronomical Telegrams, operated by Harvard University. This research has made use of the NASA/IPAC Infrared Science Archive, which is operated by the Jet Propulsion Laboratory, California Institute of Technology, under contract with the National Aeronautics and Space Administration.

\section{REFERENCES}

Abazajian, K., Adelman-McCarthy, J. K., Agüeros, M. A., et al. 2003, AJ, 126, 2081

Barucci, M. A., Capria, M. T., Coradini, A., \& Fulchignoni, M. 1987, Icarus, 72,304

Binzel, R., Morbidelli, A., Merouane, S., et al. 2010, Nature, 463, 331

Binzel, R. P., Xu, S., Bus, S. J., et al. 1993, Science, 262, 1541

Bowell, E., Chapman, C. R., Gradie, J. C., Morrison, D., \& Zellner, B. 1978, Icarus, 35, 313

Bus, S., \& Binzel, R. 2002a, Icarus, 158, 106

Bus, S., \& Binzel, R. 2002b, Icarus, 158, 146

Carvano, J. M., Hasselmann, P. H., Lazzaro, D., \& Mothe-Diniz, T. 2010, A\&A, 510, A43

Chapman, C. 2004, Annu. Rev. Earth Planet. Sci., 32, 539

Chapman, C., Morrison, D., \& Zellner, B. 1975, Icarus, 25, 104

Cutri, R. M., Wright, E. L., Conrow, T., et al. 2011, Explanatory Supplement to the WISE Preliminary Data Release Products, 2011 wise.rept

DeMeo, F., Binzel, R., Slivan, S., \& Bus, S. 2009, Icarus, 202, 160

Gaffey, M., Burbine, T., \& Binzel, R. 1993, Meteoritics, 28, 161

Gaffey, M. J., Cloutis, E. A., Kelley, M. S., \& Reed, K. L. 2002, in Asteroids III, Mineralogy of Asteroids, ed. W. F. Bottke, A. Cellino, P. Paolicchi, \& R. P. Binzel (Tucson, AZ: Univ. Arizona Press), 183

Harris, A. 1998, Icarus, 131, 291

Harris, A. W., \& Young, J. W. 1988, BAAS, 20, 865

Harris, A. W., Young, J. W., Contreiras, L., et al. 1989, Icarus, 81, 365

Hasselmann, P. H., Carvano, J. M., \& Lazzaro, D. 2011, SDSS-based Asteroid Taxonomy V1.0, EAR-A-I0035-5-SDSSTAX-V1.0, NASA Planetary Data System

Howell, E. S., Merenyi, E., \& Lebofsky, L. A. 1994, J. Geophys. Res. 99, 10, 847

Ivezić, Ž., Lupton, R. H., Jurić, M., et al. 2002, AJ, 124, 2943

Ivezić, Ž., Tabachnik, S., Rafikov, R., et al. 2001, AJ, 122, 2749

Jurić, M., Ivezić, Ž., Lupton, R. H., et al. 2002, AJ, 124, 1776

Lazarro, D., Angeli, C., Carvano, J., et al. 2004, Icarus, 172, 179

Lebofsky, L., \& Spencer, J. 1989, in Asteroids II, Radiometry and Thermal Modeling of Asteroids, ed. R. P. Binzel, T. Gehrels, \& M. S. Matthews (Tucson, AZ: Univ. Arizona Press), 128

Lebofsky, L., Veeder, G., Lebofsky, M., \& Matson, D. 1978, Icarus, 35, 336

Liu, F., Cutri, R., Greanias, G., et al. 2008, Proc. SPIE, 7017, 16

Mainzer, A., Bauer, J., Grav, T., et al. 2011a, ApJ, 731, 53

Mainzer, A., Eisenhardt, P., Wright, E. L., et al. 2005, Proc. SPIE, 5899, 262

Mainzer, A., Grav, T., Bauer, J., et al. 2011b, ApJ, accepted

Mainzer, A., Grav, T., Masiero, J., et al. 2011c, ApJ, 736, 100

Mainzer, A., Grav, T., Masiero, J., et al. 2011d, ApJ, 737, L9 
Mainzer, A., Grav, T., Masiero, J., et al. 2011e, ApJ, 741, 90

Masiero, J. R., Mainzer, A. K., Grav, T., et al. 2011, ApJ, 741, 68

Nesvorný, D. 2010, Nesvorný HCM Asteroid Families V1.0, EAR-AVARGBDET-5-NESVORNYFAM-V1.0, NASA Planetary Data System

Nesvorný, D., Jedicke, R., Whiteley, R. J., \& Ivezić, Ž. 2005, Icarus, 173, 132

Parker, A., Ivezić, Ž., Jurić, M., et al. 2008, Icarus, 198, 138

Stoughton, C., Lupton, R. H., Bernardi, M., et al. 2002, AJ, 123, 485

Tedesco, E. F., Matson, D., \& Veeder, G. 1989a, in Asteroids II, Classification of IRAS Asteroids, ed. R. P. Binzel, T. Gehrels, \& M. S. Matthews (Tucson, AZ: Univ. Arizona Press), 290
Tedesco, E. F., Noah, P., Noah, M., \& Price, S. 2002, AJ, 123, 1056

Tedesco, E. F., Williams, J. G., Matson, D. L., et al. 1989b, AJ, 97, 580

Tholen, D. 1984, Ph.D. thesis Univ. Arizona, Tucson

Tholen, D., \& Barucci, M. A. 1989, in Asteroids II, Asteroid Taxonomy, ed. R. P. Binzel, T. Gehrels, \& M. S. Matthews (Tucson, AZ: Univ. Arizona Press), 298

Veeder, G., Hanner, M. S., Matson, D. L., et al. 1989, AJ, 97, 1211

Wright, E. L., Eisenhardt, P. R. M., Mainzer, A. K., et al. 2010, AJ, 140, 1868

Xu, S., Binzel, R., Burbine, T., \& Bus, S. 1995, Icarus, 115, 1

Zellner, B., Tholen, D., \& Tedesco, E. 1985, Icarus, 61, 355 